\title{
Selbstbestimmung in einer Welt der Netze
}

Informationen aller Art sind heutzutage ein entscheidender Wirtschaftsfaktor. Dabei beschränkt sich das Interesse eines Unternehmens schon lange nicht mehr nur auf den Namen des Kunden, dessen Adresse sowie seine Kontonummer, vielmehr sind zur Marktforschung und Verbesserung der Geschäftsprozesse inzwischen alle Informationen rund um einen Menschen von Belang (Einkaufs- und Surfgewohnheiten, Aufenthaltsorte, Verbindungen zu anderen Personen in sozialen Netzen, usw.). Aufgrund der weltweiten Vernetzung sind diese Daten problemlos miteinander in Verbindung zu bringen bzw. neuen Auswertungen zuzuführen. Dabei wird häufig übersehen, dass das Recht der informationellen Selbstbestimmung die grundsätzliche Befugnis des Einzelnen darstellt, selbst über die Preisgabe und Verwendung seiner persönlichen Daten zu bestimmen. Seit das Bundesverfassungsgericht im Jahre 1983 in seinem "Volkszählungsurteil“ hierüber entschieden hat, wird das informationelle Selbstbestimmungsrecht als fester Bestandteil des Allgemeinen Persönlichkeitsrechts (Art. 2 Abs. 1 i.V.m. Art. 1 Abs. 1 GG) betrachtet. Es soll jedem Grundrechtsträger nicht nur einen Schutz, sondern eben gerade auch den Einfluss auf und das eigene Wissen über den Umgang anderer mit den ihn betreffenden Informationen und Daten gewährleisten.

Inwieweit diese Zielsetzung in Anbetracht der immer stärker vernetzten Welt überhaupt noch erreicht werden kann, ist fraglicher als je zuvor. Die immer weiter fortschreitende Entwicklung der Informations- und Kommunikationstechnik macht es unmöglich, noch nachzuvollziehen, welche Daten über eine Person bei welcher Stelle gespeichert sind. Noch weniger lässt sich feststellen, zu welchen Zwecken die Daten genutzt oder ob sie an Dritte übermittelt werden. Die zahlreichen Datenskandale der letzten Monate haben aufgezeigt, dass Datenschutz-Compliance weder bei öffentlichen Stellen, noch in Wirtschaftsunternehmen wirklich gelebt wird. Es entsteht nicht der Eindruck, dass der unzulässige Umgang mit Daten und die „Datensammelwut" wirklich mit den Novellen von 2009 gebremst wurden. Das Prinzip der Datensparsamkeit und Datenvermeidung beschreibt vielmehr einen hehren Wunsch, ist aber keine Realität. Beispiele dafür gibt es in der Praxis viele.

Nicht nur beim Zensus 2011 bestehen daher Zweifel, dass das Recht auf informationelle Selbstbestimmung verfassungsadäquat beachtet wird. Auch die Videoüberwachung des öffentlichen Raums kann einen wesentlichen Eingriff in dieses verfassungsrechtlich geschützte Recht darstellen, etwa dann, wenn sie in sensiblen Bereichen erfolgt. Es gilt daher, klare Anforderungen an die Zulässigkeit derartiger Videoüberwachungsanlagen zu finden, mit denen in der Praxis gearbeitet werden kann. Und nicht zuletzt ist auch der Bereich des Beschäftigtendatenschutzes weiterhin nicht ausreichend geregelt, und die angekündigten Reformen lassen auf sich warten, so dass sich hier ebenfalls eine Vielzahl an ungeklärten Fragen zum Schutz der informationellen Selbstbestimmung stellen. Die Beiträge dieses Heftes greifen nur einige der vielen Zweifel und ungeklärten Fragen auf und versuchen zumindest zukünftige Lösungsmöglichkeiten zum tatsächlichen Schutz der informationellen Selbstbestimmung aufzuzeigen.

\section{Britta Alexandra Mester}

